\title{
DESENVOLVIMENTO DE BANCO DE DADOS DE ÓLEOS VEGETAIS PARA APLICAÇÃO EM PROJETO DE PRODUTOS
}

\author{
C. C. OLIVEIRA ${ }^{1}$, M. TELES DOS SANTOS ${ }^{2}$ e G. A. C. LE ROUX ${ }^{2}$ \\ ${ }^{1}$ Universidade de São Paulo, Instituto de Matemática e Estatística \\ ${ }^{2}$ Universidade de São Paulo, Departamento de Engenharia Química \\ E-mail para contato: moises.teles@usp.br
}

\begin{abstract}
RESUMO - O Brasil é um país privilegiado em termos de disponibilidade de oleaginosas (clássicas ou não convencionais) com potencial de uso industrial. Integrando ferramentas computacionais aos esforços de modelagem no setor de óleos vegetais, o presente trabalho apresenta o desenvolvimento de um banco de dados de tais matérias-primas. O objetivo é reunir, integrar e disponibilizar dados físicoquímicos e econômicos para posterior integração e utilização em ferramentas de modelagem e simulação com foco em projeto de produtos. O desenvolvimento das ferramentas computacionais é descrito usando elementos de Unified Modeling Language (UML) e o banco de dados é desenvolvido através da tecnologia SQLite.
\end{abstract}

\section{INTRODUÇÃO}

O banco de dados e a respectiva interface desenvolvida, descritos nesse trabalho, foram idealizados para servir de apoio ao projeto de produtos utilizando óleos vegetais. Tais matériasprimas estão se tornando cada vez mais relevantes no cenário nacional devido à grande biodiversidade do Brasil em termos de oleaginosas e seus múltiplos usos industriais, além da utilização para produção de biocombustível (Bergman et al, 2013).

O uso de matérias-primas renováveis é essencial para o desenvolvimento sustentável na indústria química. Atualmente, há diversos incentivos do setor público, privado e acadêmico para atividades industriais sustentáveis. Óleos vegetais são matérias-primas que atendem a este critério de sustentabilidade e são usados atualmente na indústria de alimentos, lubrificantes, fluidos de corte e solventes (Teles et al, 2014), assim como em matrizes lipídicas nas quais cosméticos e princípios farmacológicos ativos são adicionados. Além dos usos clássicos, novos produtos e aplicações estão sendo estudados mundialmente, seja utilizando diretamente os óleos vegetais, seja modificando sua composição por reações químicas ou ainda utilizando o glicerol como precursor de moléculas de alto valor agregado.

A utilização de óleos vegetais para a produção de combustíveis é expressiva e ha projeções de crescimento; no entanto, a maior parte do mercado de oleos vegetais ainda é dominada por outros setores, como podemos ver na Figura 1: 


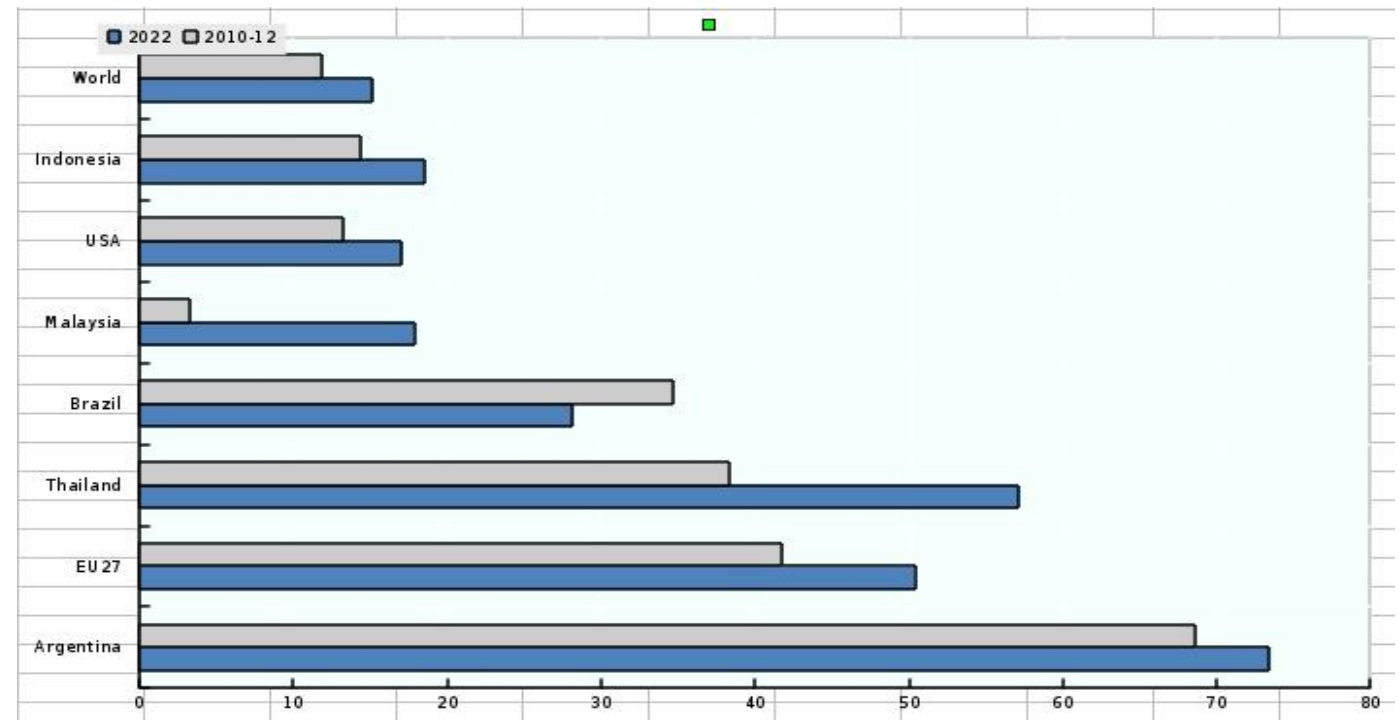

Figura 1 - Percentual de uso de óleos vegetais para produção de biocombustíveis. Fonte: OECDFAO (2013).

O objetivo do trabalho é desenvolver um banco de dados offline com a finalidade de estocar, gerenciar e utilizar o conhecimento das propriedades físico-químicas de tais produtos. $\mathrm{O}$ banco de dados guarda registros de mais de 100 propriedades para cada óleo vegetal inserido, dentre as quais encontram-se muitas essenciais para o desenvolvimento de produtos (conteúdo de gordura sólida, viscosidade, etc.). Um dos desafios do desenvolvimento dessa base de dados se encontra no fato de que as informações sobre essas propriedades estão dispersas entre vários estudos e fontes de dados experimentais. As informações dos 14 óleos mais comuns no mercado mundial foram obtidas de Shahidi (2005), mas outras fontes também serão utilizadas para constante atualização dos dados.

Esforços de uso de ferramentas computacionais para criação de banco de dados das matérias-primas nacionais de valor agregado também podem ser vistas no trabalho de Valli et al (2013). Estes autores propõem um banco de dados on-line, que insere-se no contexto de uso de ferramentas computacionais para valorização da biomassa nacional em projeto de produtos.

Para a possível comunicação entre programas que se inserem nesse mesmo contexto, associações como a CO-LaN (http://www.colan.org/) promovem o uso de padrões na fase de desenvolvimento, visando melhor interoperabilidade entre diversos programas. Os padrões de interface CAPE-OPEN, mantidos pela CO-LaN, serão adotados em futuras etapas de desenvolvimento do projeto. Por exemplo, para nomear propriedades físico-químicas, formatos de arquivos de entrada e saída, etc.

A implementação do banco de dados aqui apresentado é inspirada na avaliação dinâmica de dados descrita no trabalho de Frenkel (2011), a fim de obter resultados de projeto de produto que usem dados atualizados sempre que alguma informação nova está disponível. Frenkel (2011) dá ênfase à importância do projeto de produtos em diversas áreas nos últimos anos, e em muitas delas 
é essencial que se tenha um banco de dados atualizado para auxiliar o bom uso de uma quantidade crescente de dados experimentais.

No presente trabalho, seguem descrições dos diagramas que foram utilizados para o desenvolvimento do projeto, justificativas de escolha entre diferentes tecnologias em relação à administração do banco de dados e algumas interfaces do programa.

\section{METODOLOGIA}

Diversas tecnologias foram utilizadas para a modelagem e o desenvolvimento do projeto, das quais seguem abaixo as mais importantes, além de discussões sobre suas escolhas e como elas influenciaram o trabalho.

\subsection{Unified Modeling Language (UML)}

A modelagem do projeto foi feita com o uso da UML (Unified Modeling Language), um padrão de-facto na área de modelagem de sistemas, objetivando o estudo dos casos de uso em cada uma das possíveis utilidades do software. Essa ferramenta ajuda a ter uma visão mais ampla do projeto antes dele sair do papel e ir para a fase da programação, facilitando a implementação do sistema. A Figura 2 mostra o diagrama de casos de uso referente ao trabalho, desde a entrada e saída de informações do banco até a manipulação desses dados. Ela se divide em dois casos de uso principais descritos abaixo:

Query database: É onde entram os casos de consulta, incluindo recuperar informações de um óleo vegetal através de seu nome ou sinônimos até procurar óleos de acordo com suas propriedades fisico-químicas.

Modify database: É onde ocorre uma interação da qual resulta uma modificação no banco de dados do programa (os registros default do banco não podem ser alterados). Suas generalizações são simples: alterar, remover e inserir, onde a última opção pode ser feita de duas formas (através de um arquivo $X M L$ ou através da interface gráfica do próprio programa). É importante que o usuário possa inserir seu próprio registro de um óleo vegetal porque existem diferenças entre óleos vegetais de mesmo nome de acordo com fatores geográficos de sua região de origem. Esse registro customizado contém um campo identificador para representar diferenças entre ele e o óleo padrão de mesmo nome no banco de dados, facilitando o uso de óleos "próprios" do usuário. 


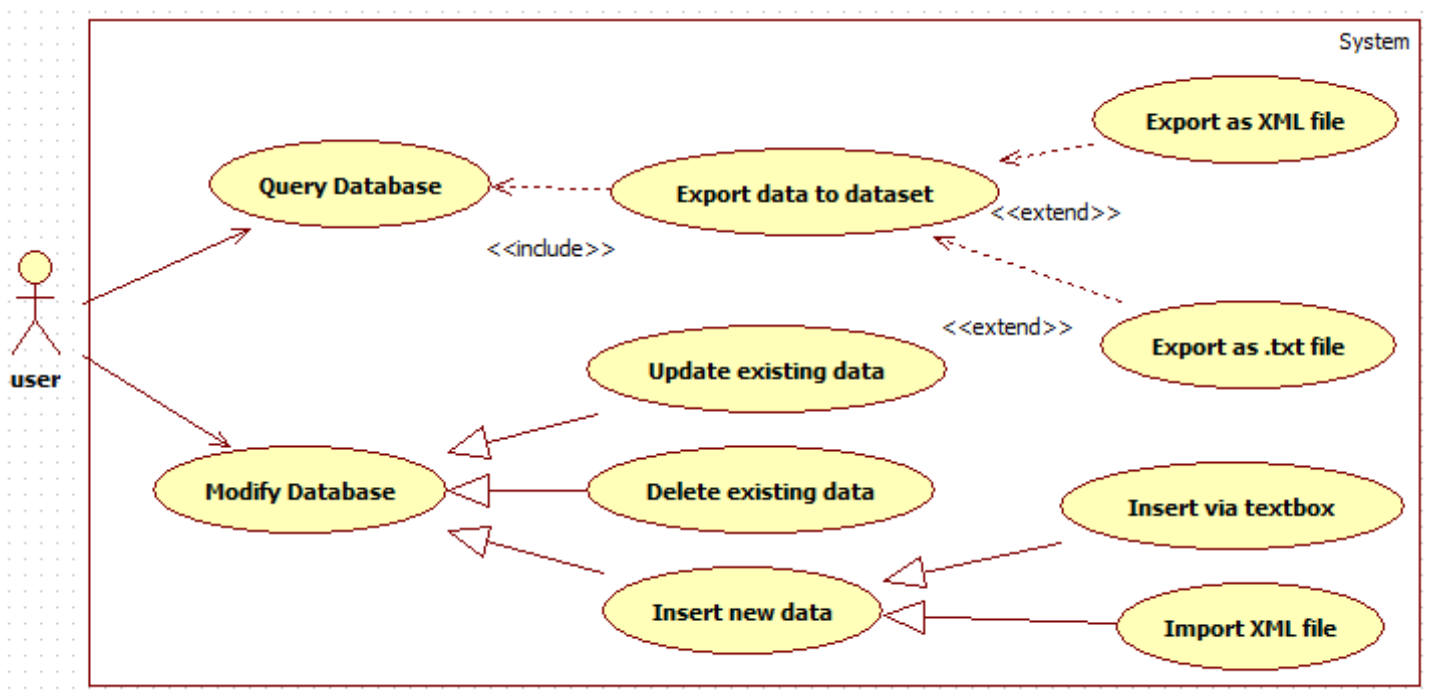

Figura 2 - Diagrama de casos de uso do banco de dados.

\subsection{Sqlite}

Para a tecnologia de administração e armazenamento dos dados foi escolhido o SQLite (http://sqlite.org), depois da análise de algumas de suas características e como elas influenciam o desenvolvimento do trabalho. Seguem algumas das características dessa tecnologia e uma breve descrição de como ela pôde ser conveniente ao projeto:

Fácil configuração: $\mathrm{O}$ banco de dados inteiro é apenas um arquivo .s3db anexado ao projeto com poucas linhas de código e através de uma classe auxiliar.

É gratuito e está no domínio público: Seu código fonte está em domínio público, portanto é livre para qualquer tipo de uso, comercial ou não.

Adequado para ser anexado em uma aplicação: Ou seja, é “instalado" junto com o projeto no momento do download (ou transferência offline) e funciona bem sem suporte técnico.

Não tem sistema de administração multi-usuário: O projeto de design de produtos no qual o banco de dados inicialmente vai ser acoplado possui um só usuário, portanto essa limitação não é um problema.

Não funciona muito bem com muitos dados: A quantidade de dados com a qual o banco trabalha não é pequena mas está longe das limitações do SQLite (http://sqlite.org/limits.html).

Não dá suporte para alta concurrência: Não há problema com essa limitação pois o banco é praticamente read-only e os poucos casos de uso onde o banco de dados é modificado não há concurrência, ou seja, só um usuário está atualizando o banco de dados por vez.

Algumas possíveis alternativas ao SQLite foram consideradas como o MySQL, que também é gratuito mas traria maiores dificuldades de configuração e apesar de ser adequado para grandes quantidades de dados, isso não era uma preocupação no projeto. Também foi considerado o PostgreSQL, que também suporta uma grande quantidade de dados e é gratuito, e tem o diferencial 
de ter um bom suporte online mas foi decidido que o banco seria offline e anexado a outras aplicações.

\subsection{C\#}

O C\# (C Sharp) é uma linguagem de programação orientada a objetos desenvolvida pela Microsoft como parte da plataforma .NET. Foi escolhida como a linguagem do projeto porque possui várias classes que dão suporte às outras tecnologias utilizadas (como o próprio SQLite, por exemplo). Também possui suporte da IDE (ambiente de desenvolvimento) da Microsoft. Essa IDE (Visual Studio), possui uma interface amigável para o desenvolvimento de aplicações visuais, o que facilita a implementação de sistemas como o apresentado no presente trabalho.

\section{RESULTADOS}

Abaixo seguem interfaces do programa que fazem parte do caso de uso Query Database, como visto na Figura 2. A Figura 3 mostra a tela onde o usuário organiza a consulta. Neste exemplo, temos uma busca que resulte em qualquer óleo com o string "flower" no meio do nome ou de algum de seus sinônimos, que contenha entre $2 \%$ e $10 \%$ de fosfolipídios e que contenha (em qualquer quantidade) ácido láurico (Lau 12:0) em sua composição. Nota-se que é possível que alguma informação não conste no banco de dados, logo aquela informação não é exibida na tela de resultados. $\mathrm{O}$ painel Results permite que o usuário clique em outro óleo resultante para que suas informações sejam carregadas na tela. Já a Figura 4 mostra a tela dos resultados (que aparece após clicarmos no botão Search mostrado na Figura 3) de uma busca com múltiplos resultados.

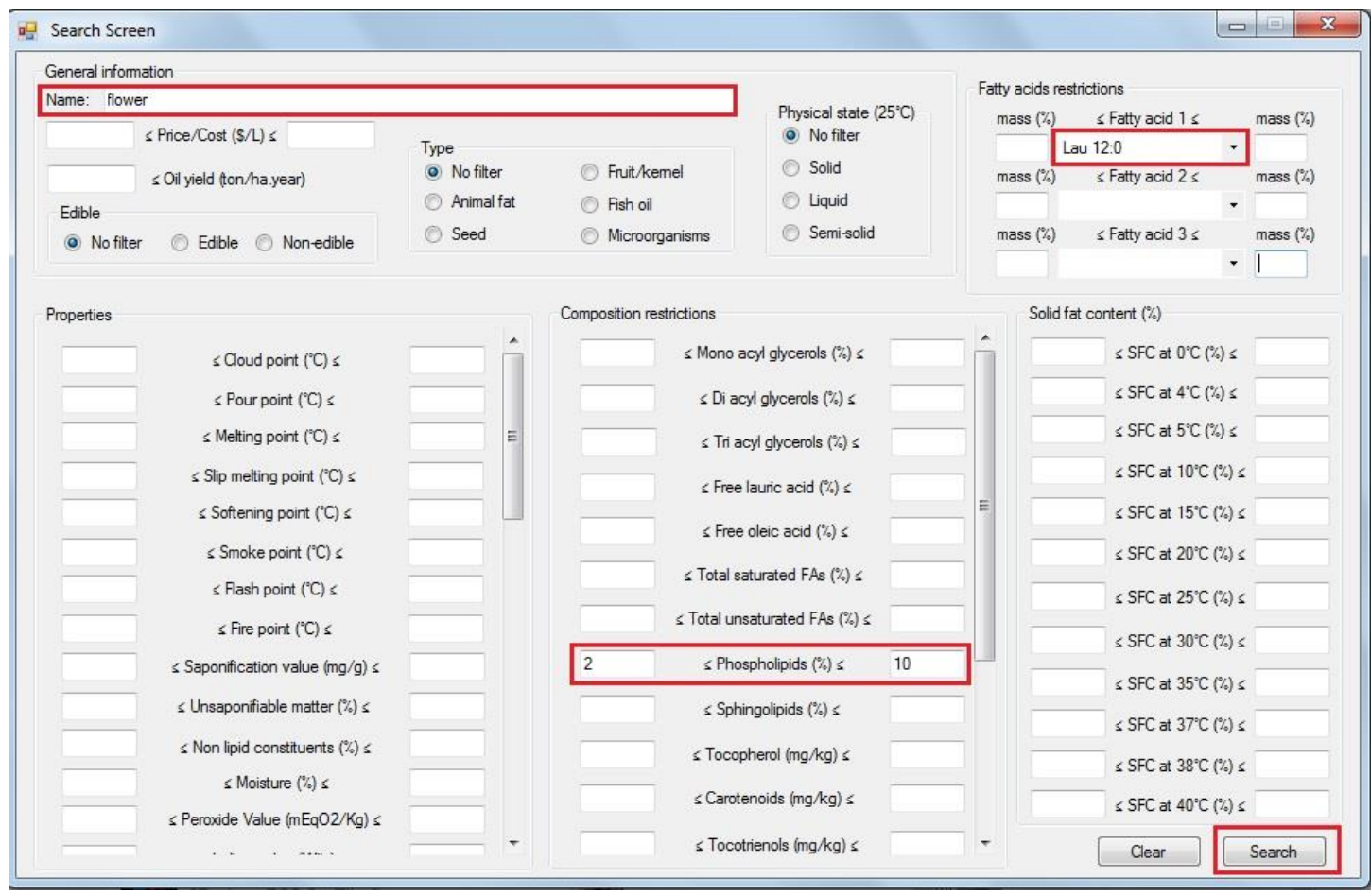

Figura 3 - Tela de busca da interface gráfica. 


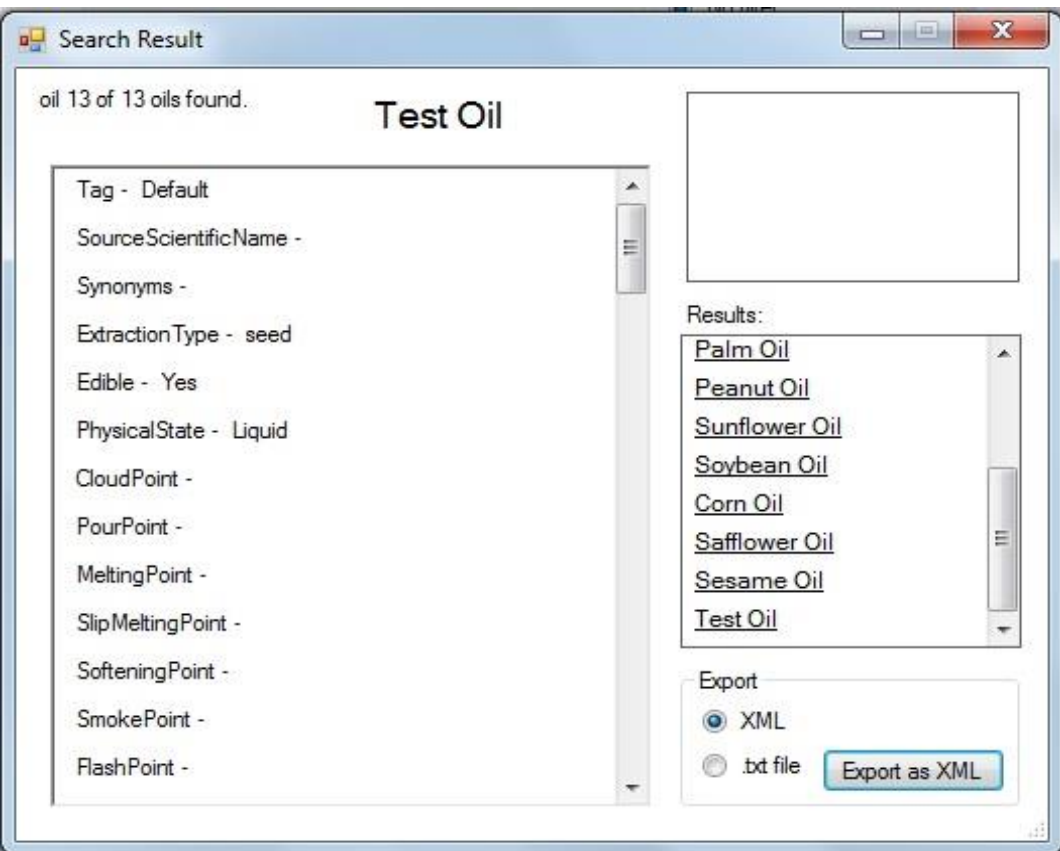

Figura 4 - Tela de resultado.

A Figura 5, a seguir, representa o caso de uso Modify Database em uma de suas generalizações: Insert new data. Esse caso de uso é importante dentro do projeto pois o usuário muita vezes decide trabalhar com óleos próprios em suas pesquisas então o programa permite que ele os acrescente com um campo identificador "TAG".

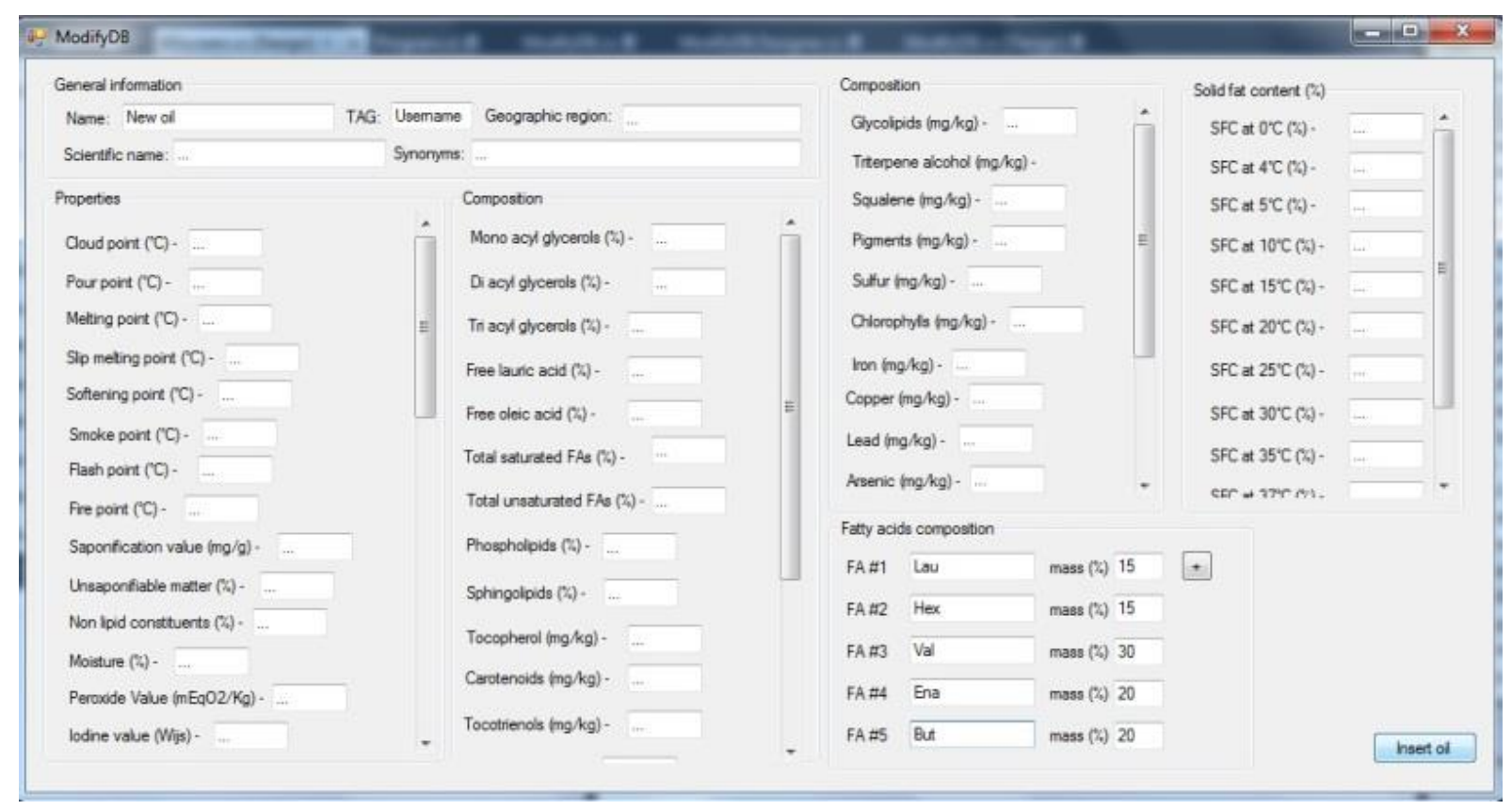

Figura 5 - Tela de inserção de novo óleo. 


\section{CONCLUSÃO}

O trabalho apresenta etapas do desenvolvimento de um banco de dados de óleos vegetais com o objetivo de selecionar matérias-primas de acordo com propriedades pré-definidas. Futuros trabalhos incluem inventariar e adicionar as propriedades físico-químicas disponíveis de todas as oleaginosas conhecidas e integrar o banco de dados a um ambiente de projeto de produtos computacional onde misturas de óleos possam ser simuladas.

\section{REFERÊNCIAS}

BERGMANN, J.C.; TUPINAMBÁ, D.D.; COSTA, O.Y.A.; ALMEIDA, J.R.M.; BARRETO, C.C.; QUIRINO, B.F.; Biodiesel production in Brazil and alternative biomass feedstocks, Renewable and Sustainable Energy Reviews, v. 21, p 411-420, 2013.

FRENKEL, M.; Thermophysical and thermochemical properties on-demand for chemical process and product design. Computers and Chemical Engineering, v. 35, p 393-402, 2011.

SHAHIDI, F.; Bailey's Industrial Oil and Fat Products. $6^{\text {th }}$ edition, v. 2, 2005.

TELES DOS SANTOS, M.; GERBAUD, V.; LE ROUX, G.A.C.; Beyond Biofuels: Opportunities and challenges for vegetable oils in the chemical industry. GPE $-4^{\text {th }}$ International Congress on Green Process Engineering, Sevilla (Spain), 2014.

VALLI, M.; DOS SANTOS, R.N.; FIGUEIRA, L.D.; NAKAJIMA, C.H.; CASTRO-GAMBOA, I.;A.D.; BOLZANI, V.S. Development of a Natural Products Database from the Biodiversity of Brazil. J. Nat. Prod., v. 76 (3), p 439-444, 2013. 